\title{
Informative gene selection using the Algebraic Connectivity Strength of Point and Scoring Criteria
}

\author{
WANG Nian ${ }^{1,2^{*}}$, SU LiangLiang ${ }^{1,2}$, TANG Jun $^{1,2} \&$ YE AiXia $^{1,2}$ \\ ${ }^{1}$ Key Laboratory of Intelligent Computing \& Signal Processing, Ministry of Education, Anhui University, Hefei 230039, China; \\ ${ }^{2}$ School of Electronics and Information Engineering, Anhui University, Hefei 230039, China
}

Received January 20, 2012; accepted March 14, 2012; published online September 21, 2012

\begin{abstract}
This paper presents a novel method that integrates the Algebraic Connectivity Strength of Point (ACSP) and Scoring Criteria to identify genes associated with tumor type. First, for each gene, the ACSP is used to identify reliable expression levels of the gene in all the samples. The informative genes are then selected using Scoring Criteria based on these reliable expression levels. Finally, the Support Vector Machine (SVM) classifier is used to classify the two datasets of gene expression profiling. The results show that the informative genes selected by the proposed method have higher credibility than those selected by Scoring Criteria alone.
\end{abstract}

tumor, gene expression profiling, Support Vector Machine, Algebraic Connectivity Strength of Point

Citation: Wang N, Su L L, Tang J, et al. Informative gene selection using the Algebraic Connectivity Strength of Point and Scoring Criteria. Chin Sci Bull, 2013, 58: 657-661, doi: 10.1007/s11434-012-5421-7

Studies based on gene expression profiling have become the mainstay of research on various life phenomena, among which the diagnosis and treatment of cancer is a research focus $[1,2]$.

However, studies based on gene expression profiling still face many challenges, such as background noise, small samples, and the dimensionality curse. To solve these challenges, researchers have proposed many methods in recent decades. Dimension reduction is one of the most commonly used methods. Using the principle of "signal-to-noise ratio (SNR)" method [3], numerous dimension reduction methods have appeared that can be generally divided into two categories: comprehensive property extraction and informative genes selection. In the former category, the dimension can be reduced through linear transformation or non-linear transformation, e.g. Principal Component Analysis (PCA) [4], Nonnegative PCA [5], Independent Component Analysis (ICA) [6], and Nonnegative Matrix Factorization (NMF) [7]. However, the results often lack a reasonable biological

*Corresponding author (email: wn_xlb@ahu.edu.cn) explanation. The methods in the latter category, which can identify informative genes with good biological explanation, are readily accepted by oncologists. These methods include the $T$ _test [8], Revised Feature Score Criterion (RFSC) [9], Relative Entropy (RE) [10], Enrichment Score [11], improved global normalized SNR (improved gn_SNR) [12], and gene regulation probability [13].

Regarding small samples, many methods based on Scoring Criteria, which utilize the mean and variance of samples, can be influenced by outliers caused by abnormal expression of genes or other reasons. It can also lead to low credibility of informative genes. To solve this problem, we propose a novel gene selection method that integrates the ACSP and Scoring Criteria to select informative genes. This method improves the credibility of the selected genes.

\section{Materials and methods}

\subsection{Scoring Criteria}

Gene expression profiling can be expressed mathematically 
as a matrix $\boldsymbol{G}$ :

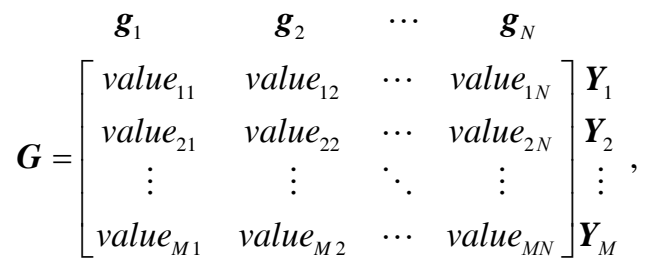

where value $_{i j}$ indicates the expression level of gene $\boldsymbol{g}_{j}$ in sample $\boldsymbol{Y}_{i}, N$ is the size of the gene and $M$ is the size of the sample, respectively. Usually $M<<N$.

There are many methods available for studying Scoring Criteria. This paper mainly focuses on $R F S C$ and $R E$ :

$$
\begin{gathered}
\boldsymbol{R} \boldsymbol{F S C}\left(\boldsymbol{g}_{i}\right)=\frac{1}{2}\left|\frac{\mu_{i,+}-\mu_{i,-}}{\sigma_{i,+}+\sigma_{i,-}}\right|+\frac{1}{2} \ln \frac{\sigma_{i,+}^{2}+\sigma_{i,-}^{2}}{2 \sigma_{i,+} \sigma_{i,-}}, \\
\boldsymbol{R E}\left(\boldsymbol{g}_{i}\right)=\frac{1}{2}\left(\frac{\sigma_{i,-}^{2}}{\sigma_{i,+}^{2}}+\frac{\sigma_{i,+}^{2}}{\sigma_{i,-}^{2}}\right)+\frac{1}{2}\left(\mu_{i,+}-\mu_{i,-}\right)^{2},
\end{gathered}
$$

here, $\mu_{i,+}$ and $\mu_{i,-}$ represent the mean of the $i$ th gene expression level in "+" category and "_" category, respectively. Similarly, $\sigma_{i,+}$ and $\sigma_{i,-}$ indicate the variance. Each gene has a similar expression level in the gene expression profiling in the same category. Nevertheless, various factors from the environment, equipment and artificial operation etc., will lead to apparent anomalies of the gene expression level in some samples. Thus, the expression levels of the gene in such samples are judged as outliers. Scoring Criteria shows good performance in obtaining informative genes, on the condition that gene expression is not contaminated. However, the mean and variance of gene expression are sensitive to outliers when the above factors exist in gene expression profiling. This undoubtedly influences the true expression and fluctuation level of genes in a given category, and subsequently lowers the credibility of the selected genes. Therefore, these outliers should be excluded for subsequent analysis.

\subsection{A novel gene selection method integrating the ACSP and Scoring Criteria}

In this paper, for a given gene, all expression levels in the same category will be considered as points on the graph. Connectivity strength is then computed using ACSP between each point and its $K$ neighbor points to determine a set of points that can best represent the true expression level of the gene in the category. Finally, outliers can be excluded for the scored gene using Scoring Criteria.

The ACSP is defined as follows:

Definition 1: Suppose a complete graph $F=\{V(F), E(F)\}$, where $V(F)=\left\{v_{1}, v_{2}, \cdots, v_{N}\right\}$ is a point set of size $N$ and $E(F)=\left\{e_{i, j}\right\}$ is an edge set. Edge $e_{i, j}$ is given with the weight $w_{i, j}, i, j \in\{1,2, \cdots, N\}$. For each point $v_{i}$, calculate the sum of $K$ adjacent edge weights of $v_{i}$ : Let $\operatorname{Sum}\left(v_{i}\right)=$ $\sum_{j=1}^{\mathrm{K}} w_{i, j}, K \leqslant N$, then $\boldsymbol{S u m}\left(v_{i}\right)$ is defined as the Algebraic

Connectivity Strength of Point (ACSP).

For each gene $\boldsymbol{g}_{i}$, the points that are the expression values of $\boldsymbol{g}_{i}$ in all the samples of a category are constructed as a complete graph. The weight of an edge is defined as follows:

$$
w_{l s}= \begin{cases}\frac{1}{\| \text { value }_{i l}-\text { value }_{i s} \|} & l \neq s \quad l, s=1,2, \cdots, \text { Num }, \\ 0 & l=s\end{cases}
$$

here Num is the number of samples in a category. Each $\boldsymbol{g}_{i}$ corresponds to a point set, denoted by Value $_{i}=\left\{\right.$ value $_{1 i}$, val$u e_{2 i}, \cdots$, value $\left._{\text {Numi }}\right\}$. When the number of neighboring points $K \approx T \times N u m$, where $T(T \in[0,1])$ is a parameter, calculate $\operatorname{Sum}\left(\right.$ value $\left._{j i}\right)$ :

$$
\operatorname{Sum}\left(\text { value }_{j i}\right)=\sum_{s=1}^{\mathrm{K}} w_{j s} \quad j=1,2, \cdots, \text { Num }
$$

first, the maximum is determined, $\operatorname{Sum}_{\max }=\operatorname{Max}\left\{\operatorname{Sum}\left(\right.\right.$ value $\left._{1 i}\right)$, $\operatorname{Sum}\left(\right.$ value $\left._{2 i}\right), \cdots, \operatorname{Sum}\left(\right.$ value $\left.\left._{\text {Numi }}\right)\right\}$. Then the value $_{j i}$ corresponding to Sum $_{\max }$ is considered as the center point. The mean and variance of the expression level of gene $\boldsymbol{g}_{i}$ can be obtained in one category through analyzing $T \times N u m$ points adjacent value $_{j i}$ (including value ${ }_{j i}$ ). The mean and variance of the expression level of gene $\boldsymbol{g}_{i}$ in the other categories may be obtained in the same way. Finally, gene $\boldsymbol{g}_{i}$ is scored according to eq. (2) or (3).

\section{Experimental procedure}

Compared with the dimension, the sample size shows an extreme imbalance in gene expression profiling, i.e., few samples in a high dimensional dataset. Hence, we adopted leave-one-out cross-validation (LOOCV) to train the SVM classifier. LOOCV means randomly selecting one sample as a test sample from the entire dataset with $n$ samples, then the remaining $n-1$ samples are used to train SVM to gain the optimal decision model that determines which class the test sample belongs to. This process does not stop until all samples have been tested separately. Then, the number of test samples that were correctly classified can be determined.

In fact, the training problem of the SVM classifier is a convex quadratic programming problem, which can be transformed into seeking the optimal solution of the following objective function.

Max:

$$
\sum_{i=1}^{n} a_{i}+\frac{1}{2} \sum_{j=1}^{n} a_{i} a_{j} y_{i} y_{j} K\left(x_{i}, x_{j}\right),
$$

Subject to:

$$
\left\{\begin{array}{c}
0 \leqslant a_{i} \leqslant C \quad i=0,1, \cdots, n \\
\sum_{i=1}^{n} y_{i} a_{i}=0
\end{array},\right.
$$


where $a_{i}$ is the Lagrange Multiplier, $y_{i} \in\{1,-1\}$ is a category label of sample $x_{i}$, and $C$ is the upper boundary of the Lagrange Multiplier. The decision function,

$$
f(x)=\operatorname{sign}\left(\sum_{i=1}^{n} a_{i} y_{i} K\left(x_{i}, x\right)+\mathrm{b}\right),
$$

is then used to determine which category sample $x$ belongs to. Here $\mathrm{b}$ is a constant and $K\left(x_{i}, x\right)=\exp \left(-\left\|x-x_{i}\right\|^{2} / \sigma^{2}\right)$. In this paper, the parameter: $C=200$ and $\sigma=7$ from a leukemia experiment $(\sigma=3$ in the diffuse large B cell lymphoma (DLBCL) experiment), were determined when the number of the test samples which were correctly classified was the largest. The experimental steps are as follows:

Step 1: In a given category $\boldsymbol{C}_{k}$, for gene $\boldsymbol{g}_{i}$, calculate the mean and variance in two categories using the ACSP, respectively. Gene $\boldsymbol{g}_{i}$ is scored according to the Scoring Criteria by eqs. (2) and (3).

Step 2: Similar to Step 1, all the genes will be scored and ranked in a descending order.

Step 3: Select the first $L$ genes as the informative genes subset, and perform LOOCV experiments using SVM.

Step 4: Locate subsequently informative genes based on the experimental results. To assist oncologists to prevent and treat cancer, provide the location of these genes.

\section{Results and analysis}

This paper used two groups of public datasets: a Leukemia dataset and the DLBCL dataset. The leukemia dataset consists of 52 samples with 12564 genes in each sample. The samples include two subtypes: 24 Acute Lymphoblastic Leukemia (ALL) samples and 28 Acute Myeloid Leukemia (AML) samples (http://www.broadinstitute.org/cgi-bin/cancer/ datasets.cgi). DLBCL comprises 19 follicular lymphoma (FL) samples and 58 DLBCL samples with each sample containing 5469 genes (http://www.gems-system.org/). The description of all the genes and more information is available in the corresponding websites. The two datasets were normalized in this paper.

\subsection{Leukemia dataset}

From the perspective of a small-scale analysis of genes, the classification accuracy of ACSP+RFSC is significantly higher than that of RFSC alone when $L<20$ (Figure 1). Under the condition of $L=3$, the classification accuracy of this method achieves $98.8 \%$, while classification accuracy of RFSC alone is less than $20 \%$. This is probably because several of the most important genes, which had been contaminated, are selected by RFSC as informative genes. Nevertheless, this can be avoided by ACSP+RFSC.

In Figure 2, gene No. 3634 obtained by ACSP+RFSC has higher expression levels in the ALL category than in the

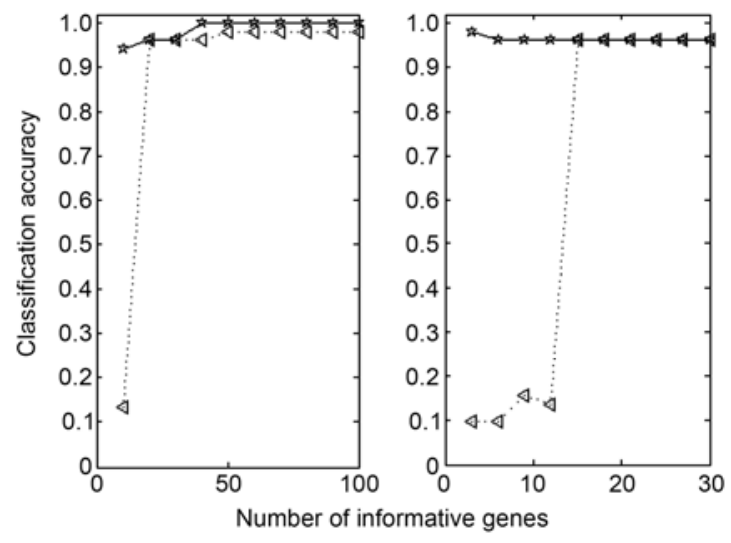

Figure 1 Classification results of the Leukemia dataset with ACSP+ $\operatorname{RFSC}(\lesssim)$ and $\operatorname{RFSC}(\nabla)$, respectively.

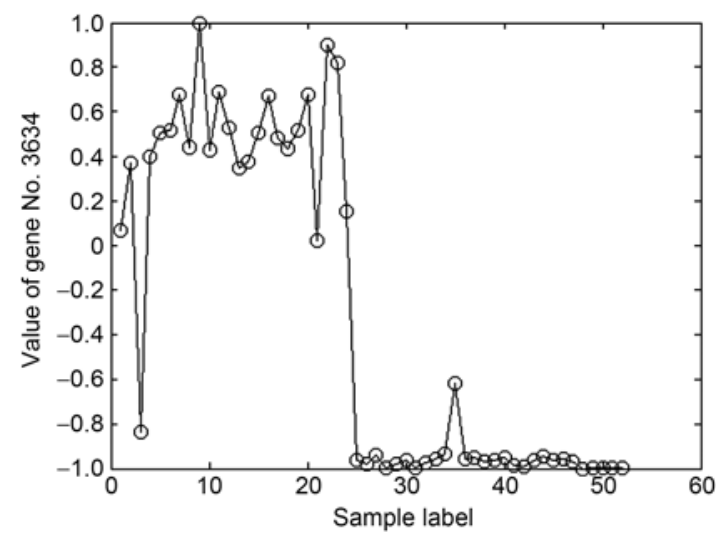

Figure 2 Expression levels of gene No. 3634 with highest scores by ACSP+RFSC in all the samples (sample label: 1-24, ALL samples; 25-52, AML samples).

AML category, and almost all expression levels with large fluctuations are greater than 0 in the ALL category. On the other hand, the expression values are close to -1 and the expression level is stable in the AML category. Apparently, gene No. 3634 is a very good discriminant factor to determine which category a sample belongs to. However, we noticed that 23 expression levels of gene No. 282 in the AML category are similar to those in the ALL category (Figure 3). Only five distinctly different expression levels as outliers lead to the mean and variance of the expression level of gene No. 282 being seriously affected. Therefore, gene No. 282 may be assumed to be a factor with no discriminatory ability.

The influence of these outliers can be avoided when neighboring points $K \approx T \times N u m$ (AML: $22.4=0.8 \times 28$, if $T=0.8$ ), such that the five outliers are excluded. The mean and variance obtained can better reflect the real situation of gene expression and improve the credibility of the genes as informative genes.

To further illustrate the advantages of ACSP+RFSC, 50 genes selected are shown (Figures 4 and 5). There are 


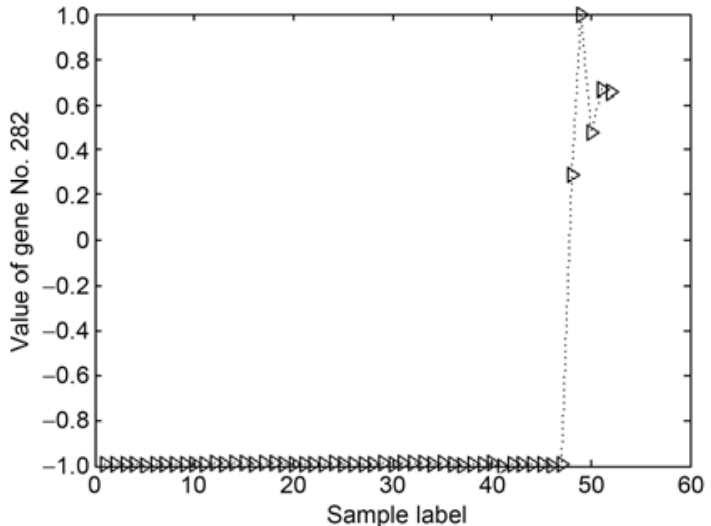

Figure 3 Expression levels of gene No. 282 with highest scores by RFSC in all the samples (sample label: 1-24, ALL samples; 25-52, AML samples).

significant blocks in Figure 4 showing that the expression levels of genes in ALL samples are obviously less than those in AML samples (genes labeled 1-12), and expression levels of genes labeled 13-50 in ALL samples are greater than those in AML samples. However, using RFSC alone, most of the genes are not discriminant factors, except genes labeled as 3, 6, 34, and 36 (Figure 5). Therefore, the performance using RFSC alone is poor.

\subsection{DLBCL dataset}

The DLBCL dataset was tested to evaluate the proposed method (Figure 6). The classification accuracy of ACSP+ RFSC is higher than that of RFSC when the number of informative genes is 30 to 150 . The highest classification accuracy is $97.40 \%$ in the right part of Figure 6 . The method is superior to RFSC with respect to selecting a few of the most important genes.

We designed the method ACSP+RE to analyze the two datasets (Figure 7) and the problem of informative genes redundancy was briefly considered. The redundancy is judged according to the classification accuracy and it gradually

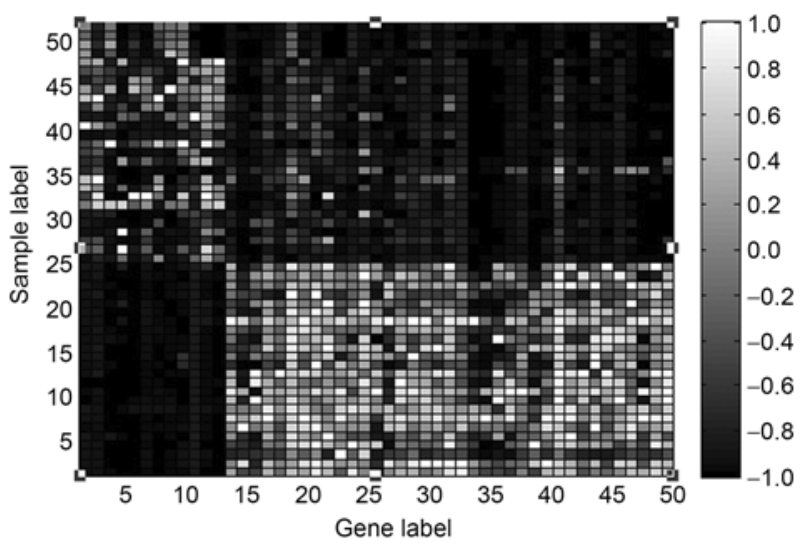

Figure 4 Expression levels of 50 informative genes with ACSP+RFSC (sample label: 1-24, ALL sample; 25-52, AML sample).

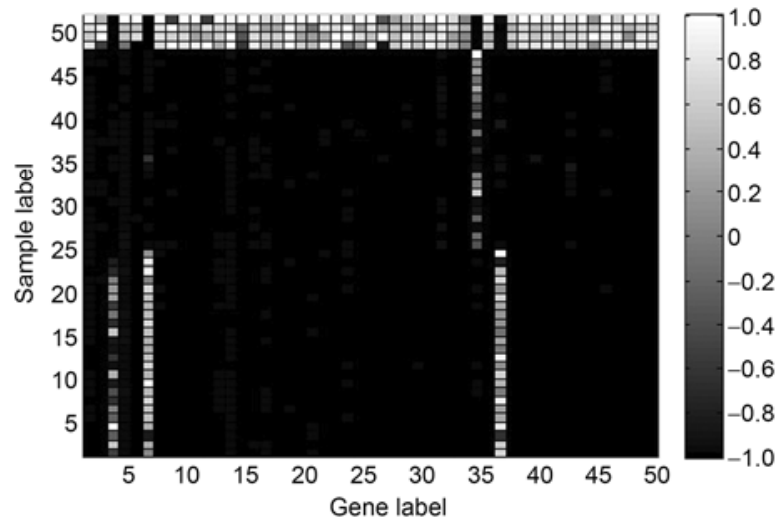

Figure 5 Expression levels of 50 informative genes with RFSC (sample label: 1-24, ALL sample; 25-52, AML sample).

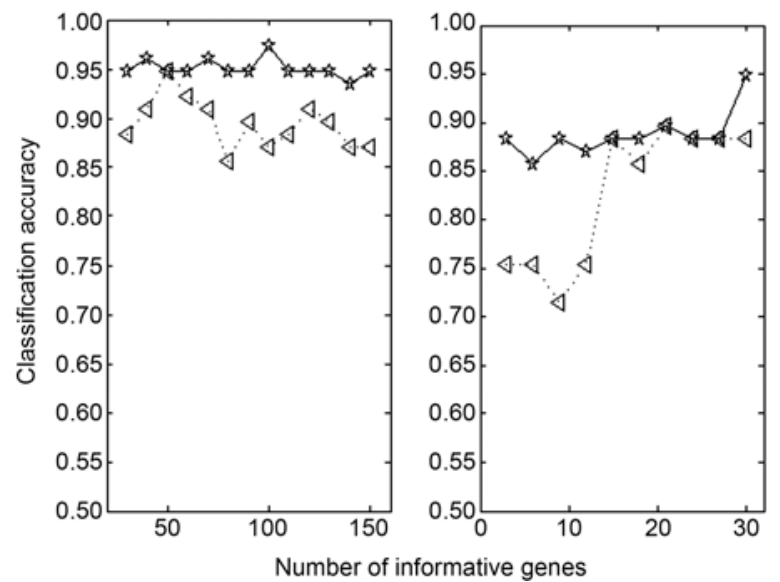

Figure 6 Classification results of the DLBCL dataset with ACSP+RFSC (ఓ) and RFSC $(\nabla)$, respectively.

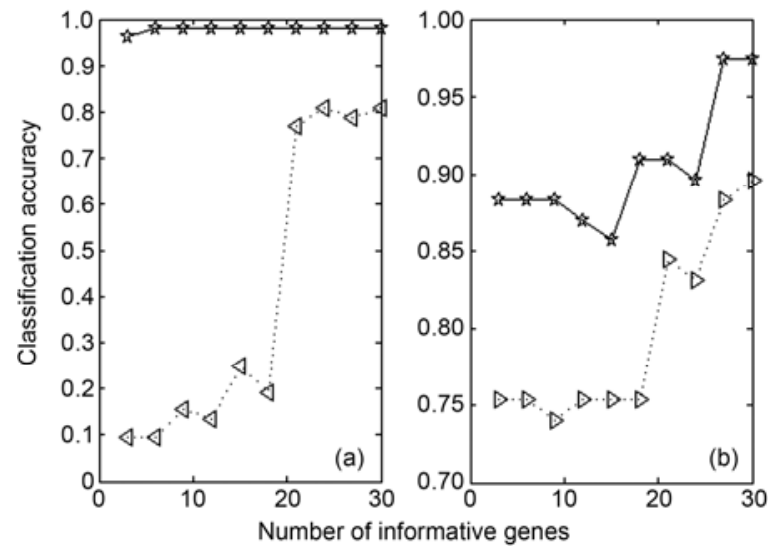

Figure 7 Classification results of the Leukemia and DLBCL datasets by $\mathrm{ACSP}+\mathrm{RE}($ 污) and RE $(\nabla)$, respectively ((a) Leukemia; (b) DLBCL).

increases with increasing numbers of selected informative genes. The classification accuracy reaches its highest value when the number of informative genes reaches a certain number. Any further increase the number of informative 
genes causes the classification accuracy to plateau, or even decline. This implies that genes selected later have produced redundant information and therefore represent noisy genes.

The above analysis shows that the informative genes obtained by the proposed method have a higher reliability. Finally, according to these experimental results, this paper has provided some genes associated with tumor type to biomedical experts, as shown in Table 1.

\section{Conclusions}

The study shows that the expression level of a gene, which is impacted by outliers, is only a small proportion in a given category. The expression level of the gene will deviate from the real situation if Scoring Criteria, which does not exclude these outliers, is used alone. On the other hand, the experimental results of the Leukemia and DLBCL datasets indicate that the developed ACSP+RE method can exclude the

Table 1 The genes associated with tumor type obtained by the ACSP+RE method from the Leukemia and DLBCL dataset, respectively ${ }^{\mathrm{a}}$

\begin{tabular}{cl||rl}
\hline \multicolumn{2}{c||}{ Leukemia dataset } & \multicolumn{2}{c}{ DLBCL dataset } \\
\hline Label & Feature & Label & \multicolumn{1}{c}{ Feature } \\
\hline 9832 & 40282_s_at & 4588 & V00594_s_at \\
8585 & 36638_at & 52 & AB002409_at \\
1119 & 34168_at & 5136 & X65965_s_at \\
3634 & 39318_at & 1600 & M57710_at \\
5627 & 36021_at & 5133 & X06700_s_at \\
12418 & 266_s_at & 3124 & X01060_at \\
3277 & 38242_at & 478 & D79997_at \\
11607 & 1077_at & 4323 & D13666_s_at \\
8869 & 37403_at & 3867 & Y08374_rna1_at \\
3399 & 38604_at & 3564 & X76534_at \\
6278 & 37988_at & 4767 & M14328_s_at \\
11603 & 1096_g_at & 3127 & X02152_at \\
9005 & 38017_at & 1055 & L17131_rna1_at \\
\hline
\end{tabular}

a) The first column and third column "Label" correspond to the location of genes in $\boldsymbol{G}$ (eq. (1)). The column "Feature" indicates the real location from which the detailed information concerning the gene can be obtained from the corresponding websites (http://www.broadinstitute.org/cgi-bin/ cancer/datasets.cgi; http://www.gems-system.org/). impact of these outliers and the real expression level of the gene can be reflected better, without analyzing these outliers. Future work will concentrate on exploring the numbers informative genes in relation to different types of tumors and the redundancy problem of informative genes.

This work was supported by the National Natural Science Foundation of China (61172127), the Anhui Provincial Natural Science Foundation (1208085MF93), and the Innovative Research Team of "211" Project of Anhui University.

1 Ghorai S, Mukherjee A, Sengupta S, et al. Cancer classification from gene expression data by NPPC ensemble. IEEE/ACM Trans Comput Biol Bioinform, 2011, 8: 659-671

2 Fang O H, Mustapha N, Sulaiman M N. Integrating biological information for feature selection in microarray data classification. 2010 Second International Conference on Computer Engineering and Applications (ICCEA), 2010, 2: 330-334

3 Golub T R, Slonim D K, Tamayo P, et al. Molecular classification of cancer: Class discovery and class prediction by gene expression monitoring. Science, 1999, 286: 531-537

4 Nishimura K, Abe K, Ishikawa S, et al. A PCA based method of gene expression visual analysis. Genome Informatics, 2003, 14: 346-347

5 Han X X. Nonnegative principal component analysis for cancer molecular pattern discovery. IEEE/ACM Trans Comput Biol Bioinform, 2010, 7: 537-549

6 Lutter D, Stadlthanner K, Theis F, et al. Analyzing gene expression profiles with ICA. In: Proceedings of the 24th IASTED International Conference on Biomedical Engineering, Innsbruck, Austria, 2006. 25-30

7 Zheng $\mathrm{C} \mathrm{H}$, Huang D S, Zhang L, et al. Tumor clustering using nonnegative matrix factorization with gene selection. IEEE Trans Inf Technol Biomed, 2009, 13: 599-607

8 Jafari P, Azuaje F. An assessment of recently published gene expression data analyses: Reporting experimental design and statistical factors. BMC Med Inform Decis Mak, 2006, 6: 27

9 Li Y X, Ruan X G. Feature selection for cancer classification based on support vector machine. Comput Res Develop, 2005, 42: 1796-1801

10 Yan X T, Deng M H, Fung W K, et al. Detecting differentially expressed genes by relative entropy. J Theor Biol, 2005, 234: 395-402

11 Revathy N, Amalraj D R. Accurate cancer classification using expressions of very few genes. Int J Comput Appl, 2011, 14: 19-22

12 Du W, Wang Y, Wang D P, et al. An improved normalized signal to noise ratio method for irrelevant genes removing. 2010 3rd International Conference on Biomedical Engineering and Informatics (BMEI), 2010, 6: 2275-2279

13 Wang H Q, Huang D S. A gene selection algorithm based on the gene regulation probability using maximal likelihood estimation. Biotechnol Lett, 2005, 27: 597-603

Open Access This article is distributed under the terms of the Creative Commons Attribution License which permits any use, distribution, and reproduction in any medium, provided the original author(s) and source are credited. 